\section{Autism spectrum disorder in a community-based sample with neurodevelopmental problems in Lagos, Nigeria}

\author{
Yewande O. Oshodi, ${ }^{1}$ \\ Andrew T. Olagunju, ${ }^{1}$ \\ Motunrayo. A. Oyelohunnu, ${ }^{1}$ \\ Elizabeth A. Campbell, ${ }^{1}$ \\ Charles S. Umeh, ${ }^{1}$ Olatunji F. Aina, ${ }^{1}$ \\ Wellington Oyibo, ${ }^{2}$ Folusho E.A. Lesi, ${ }^{3}$ \\ Joseph D. Adeyemi ${ }^{1}$ \\ Departments of ${ }^{1}$ Psychiatry, ${ }^{2}$ Medical \\ Microbiology and Parasitology, \\ ${ }^{3}$ Pediatrics, University of Lagos, Nigeria
}

\section{Abstract}

Autism Spectrum Disorder (ASD) is a globally prevalent neurodevelopmental disorder for which early diagnosis and intervention is the mainstay of management. In the African continent, limited data is available regarding the non-clinic based samples. Lack of information available to caregivers and inadequate skilled manpower often limit early detection and access to the few available though under resourced services in the community. Community based screening can be an important drive to create awareness and improve information dissemination regarding services available for those living with this disorder. This is a descriptive cross-sectional study utilizing data obtained from participants of a community-based autism screening exercise. The surveillance exercise was part of the annual Orange Ribbon initiative for autism awareness and screening held in 2014. Data was obtained from 85 participants involved in the Autism Surveillance screening exercise within the Lagos community. Community public service radio announcements state wide and word of mouth were used to invite and enroll eligible participants to the screening and consultation exercise. A second stage screening and a brief sociodemographic questionnaire followed by a third stage clinical interview and evaluation using the Diagnostic and Statistical Manual of Mental Disorders - 5 Edition (DSM 5) were used. Appropriate consultation and referrals to services in the community were given. Participants had a mean age of 7.53 years (SD 4.35). Twenty-nine (34.5\%) met the diagnosis of ASD. Other diagnosis included attention deficit hyperactivity disorder (ADHD), language and speech disorder, intellectual disability (8.3\%) and learning disorders $(9.5 \%)$. Main health concerns to caregivers were poor language development in all (100\%), of which 11 (40.7\%) were non-ver- bal; gaze avoidance was seen in 14 (48.3\%) and challenging behavior in 12 (42.9\%). Comorbidities included seizure disorders (3.4\%) and ADHD (6.9\%). Persons with autism had history of ASD behavior more often when compared to the other neurodevelopmental disorders and these findings were statistically significant. Referrals were given to caregivers to engage in services within the community. As seen in this study, community understanding of ASD is poor in such locations, in which many persons with other neurodevelopmental disorders are often presented as having autism. Caregivers in the study location are distressed by many symptoms associated with autism and their comorbid conditions. Currently there is an evident role for frequent large scale community based screening and autism awareness exercises possibly using inter-sectoral collaboration as a strategy.

\section{Introduction}

Autism Spectrum Disorder (ASD) is characterized by impairments in social communication, interaction and behavior. ASD is now defined by the American Psychiatric Association's Diagnosis and Statistical Manual of Mental Disorders - 5 edition (DSM-5) ${ }^{1}$ as a single disorder that includes disorders that were previously considered separate: autism, Asperger's syndrome, childhood disintegrative disorder and pervasive developmental disorders not otherwise specified. ASD is a globally existing neurodevelopmental condition affecting several hundreds of persons and their families. The process of diagnosing autism has been modified in 2014 with the recent changes in the DSM-5, in which the diagnosis is based on deficits in 2 main domains. These domains are namely, the social and communicative deficits, and the presence of repetitive, restrictive behaviors and interests, all existing within the early neurodevelopmental stages. It is, however, not unusual to find individuals with comorbidities or complications that further confuse the care giver as to the nature of the illness and in the absence of skilled health workforce and routine ASD screening the children may not receive an early diagnosis.

Society, culture and enlightenment however play an important role in how parents and families seek help for early diagnosis. Parental belief systems on the causative nature of the disorder have been found to be varied. Herbet and Kohenglioh ${ }^{2}$ discovered that parents believed genetics, environmental factors and events relating to child birth could contribute to the etiology. Researchers have also shown that in multi-cultural communities, Caucasian Americans were more likely to be diagnosed earlier than the other minority groups. ${ }^{3,4}$ In
Correspondence: Yewande $0 . \quad$ 0shodi, Department of Psychiatry, University of Lagos, University Rd, Lagos, Nigeria

Tel.: +277.12293011.

E-mail: yewyoshodi@yahoo.co.uk

Key words: Autism, Africa, Community, Neurodevelopmental disorders.

Contributions: All authors were involved in the Study conception and planning for data collection. Manuscript content development was by YO, EC, AO and MA. All authors also made contributions to manuscript writing and editing at different stages of its preparation. Coordination of manuscript preparation among all authors was by corresponding author.

Acknowledgments: this surveillance exercise was supported by the management of the College of Medicine University of Lagos and Guaranty Trust Bank PLC. Nigeria as part of its annual CSR "Orange ribbon" initiative for annual surveillance and community enlightenment program for ASD. Appreciation is extended to all members of the team of volunteers and Team from Blazing Trails International, Dallas,Texas USA who all assisted in the screening exercise and clinical evaluations and also to all caregivers and persons with ASD that participated in the program.

Conflict of interest: the authors declare no potential conflict of interest.

Received for publication: 7 June 2016. Accepted for publication: 1 January 2017.

This work is licensed under a Creative Commons Attribution NonCommercial 4.0 License (CC BYNC 4.0).

(C) Copyright Y.O. Oshodi et al., 2016

Licensee PAGEPress, Italy

Journal of Public Health in Africa 2016; 7:559

doi:10.4081/jphia.2016.559

addition, in the USA, black children were found to receive a diagnosis of intellectual disability more. ${ }^{5}$ In Nigeria, Bakare et $a l .{ }^{6}$ described the role of negative belief systems and cultural practices in Africa as significant in contributing to delays in seeking for care. Bakare et al. ${ }^{6}$ observed that the etiological basis of ASD is still being explained by supernatural causes in Africa and that individuals with ASD and their families often face rejection, negative and derogatory comments, which further promotes stigma.

Early diagnosis and intervention remains the mainstay of ASD management, though this is yet to be successfully achieved in many African countries. The modest and relevant efforts of the African continent to create awareness in the field of ASD and to support sufferers and their families are still inadequate for the need. In Africa and other 
resource constrained settings, collaboration between sectors or organizations may present viable partnerships for such screening. Such collaborations are much needed in the face of poor human resource, services and inadequate allocation of funds needed to provide effective evidence-based models of services for detection, intervention and rehabilitation. In the field of autism care and research, the use of collaborative partnerships may serve as one of the important tools in many centers where experts can offer service; private sectors can offer funding and nongovernmental groups can volunteer skills in early detection and linkage to the sparse but avalable resources within the community. Such collaborations have been successfully implemented in other aspects of health care service delivery. ${ }^{7,8}$ In most African countries there is a dearth of experts in the field of autism research, as a result children with such diagnosis are often not identified and even when they are identified, the parents struggle with stigma and discrimination from the community and in addition they have very limited options of where to go in order to get confirmation of diagnosis or treatment. ${ }^{6}$

ASD qualifies as a condition with considerable public health significance and impact because of its impact on the sufferer and their caregivers along with the long lasting deficits in the face of inadequate human and capital resources to diagnose and treat it. The use of inter-sectoral or interagency collaboration becomes an important strategy for ensuring public health promotion regarding awareness, screening and referral for treatment of ASD.

In view of the current limited data on ASD from the African countries in general and Nigeria in particular, along with the challenges of resources and skill, collaborative efforts can be explored for regular screening and evaluation of clinic and community based samples. There is much to gain and to be derived from such partnerships such as provision of funding, increased reach of awareness, sharing of expertise, and assessments of individuals within the general population. The authors aim at determine common characteristics, comorbidities, caregiver concerns of ASD among children and adolescents with neurodevelopmental disorders from such a collaborative community based screening exercise.

\section{Materials and Methods}

\section{Study location and design}

This is a descriptive cross-sectional study that utilized data obtained from participants of the 2014 annual Autism Consultation Orange Ribbon initiative which is an annual surveillance and community enlightenment program for ASD. Organizers are multi-sectoral stakeholders collaborating in a partnership made of an expert team of mental health and allied clinical team members from the Autism Committee of the College of Medicine University of Lagos (CMUL), along with an international multidisciplinary team from Blazing Trails International (USA), a non-governmental organization. Funders were from a corporate organization, GTBank, which is a public sector organization. Surveillance activities and detailed clinical evaluation went on for two days with the clinical team working in shifts.

\section{Participants}

Participants were invited via state wide radio public service announcements in the communities along with word of mouth inviting individuals with children suspected of having autism based on the descriptions provided in the announcements. Interested participants registered their wards online or called in to indicate their interest and they were asked to describe the key features in their children and to provide some details about the condition. Eligibility criteria included: a history of onset of developmental and/or behavioral problems since early childhood; delayed milestones; poor social interaction; children between the ages of two and eighteen years of age; no specific chronic medical history; and no record of visual or hearing deficits. Eligible and willing participants proceeded to the second stage and received informed consent and these attendees then went on to a second stage face to face screening and then a clinical evaluation.

\section{Procedure}

All consecutive participants and caregivers completed relevant socio-demographic questionnaires. Initial screening was based on the MCHAT/CAST questions along with a detailed history; those suspected of having ASD went on to an evaluation by an experienced psychiatrist using the DSM 5 criteria for ASD along with a clinical interview. All participants subsequently received counseling about the needs of their children and were given direction regarding how to access available services in the community.

\section{Measures}

\section{Socio-demographic questionnaire}

This set of questions specifically designed for the study was used to obtain details of each participant. Variables such as age, gender, type of delivery, birth position, sibling health and a full medical history were obtained with full consent.

\section{The Modified Checklist for Autism in}

\section{Toddlers}

The Modified Checklist for Autism in Toddlers (M-CHAT) ${ }^{9}$ is validated for screening toddlers between 16 and 30 months of age to assess risk for ASD. The M-CHAT can be administered and scored as part of a well-child check-up and can also be used by specialists or other professionals to assess the risk for ASD. The primary goal of using the M-CHAT was to maximize the detection of ASD. However, caution was applied as there is a high false positive rate with the M-CHAT, meaning that not all children who score at risk for ASD will be diagnosed with ASD. The MCHAT can be scored in less than two minutes. Individuals with responses suggestive of ASD were further evaluated for ASD.

\section{The Childhood Autism Spectrum Test}

The Childhood Autism Spectrum Test (CAST) was formerly known as the Childhood Asperger Screening Test, but then Allison et al. ${ }^{10}$ renamed it as the Childhood Autism Spectrum Test. The change of the name reflects the fact that it was originally designed for a prevalence study of Asperger Syndrome in childhood but is useful for low-functioning children on the autism spectrum scale too. The CAST is a 39 -item, yes or no evaluation, aimed at parents. The questionnaire was developed by ARC (the Autism Research Centre) at the University of Cambridge, for assessing the severity of autism spectrum symptoms in children.

The CAST has been used in populationbased epidemiological research as a screen for autism spectrum disorders in primary school aged children, and previous studies have shown reasonable sensitivity and specificity when using a screening cut-point of $\geq 15{ }^{11}$ The CAST was used to screen older children for ASD before proceeding to have the clinical assessment if suspected to have autism.

\section{DSM-5 criteria for autism spectrum disorder}

The DSM-5 criteria released in 2013, describes 2 main domains of deficits required for a diagnosis of ASD: social communication and social interaction deficits; and restricted and repetitive patterns of behavior, interests or activities. There are specific details for each domain, but deficits in both domains must have been present in the early neurodevelopmental period. These criteria are often explored in clinical settings and a diagnosis is often made based on a thorough history and clinical evaluation.

Diagnosis of other comorbidities and neurodevelopmental disorders were based on history, clinical interviews and meeting criteria following detailed history and clinical examination. 


\section{Ethical considerations}

All primary caregivers who accompanied the participants gave written informed consent to participate willingly in the program. They were informed that the data obtained from the exercise will be published while preserving the confidentiality of their participant. This program received ethical approval from the Lagos University Teaching Hospital (LUTH), ADM/DCBT/HREC/2230. All participants identified requiring medical attention especially with regard to comorbidities were referred to the relevant professionals or specialists, while those needing interventions for different deficits were connected with resources within the community. Funders of this program had no role in the analysis of this data nor its preparation of this manuscript for publication.

\section{Analysis of data}

Data was collected at the location of the exercise and entered into a spreadsheet. Analysis was done using SPSS version 17. Descriptive analysis was carried out.

\section{Results}

A total of 85 children and adolescents aged between 2-18 years were evaluated, the age range was between 3-17 years. The mean age (standard deviation) of the children was 7.53 $( \pm 4.35)$ years. All the children were accompanied by their parents.

All participants were referred for suspected ASD. However, following the screening, a variety of diagnoses were found among the participants. A total of 19 (34.5\%) participants met the criteria for autism after the clinical interview based on the DSM-5 criteria. In some cases, more than one diagnosis was made. Some of the other diagnoses recorded among the attendees were Down's syndrome in 7 (8.3\%), ADHD in 10 (13.1\%), speech and language communicative disorders in 16 (19.0\%) (Table 1).

In the children diagnosed with autism spectrum disorder major concerns identified by the caregivers varied. Language problems were found to be of greatest concern (100\%). Some of the other common concerns that were reported included coordination problems in 12 (42.9\%), attention problems in 10 (35.7\%) and poor social skills in 6 (21.4\%). The early developmental history obtained about the children meeting the criteria for autism were as follows; almost all (92.9\%) had 9 month pregnancy with only 2 (7.1\%) being premature babies. Twenty (71.4\%) of them cried immediately at birth. A history of developmental delays was evident in different domains, with 26 (92.9\%) showing speech delays and 14 (48.3\%) poor eye gaze. Five (17.9\%) of these children had a seizure disorder comorbid with the autism diagnosis (Table 2).

Twenty three (79.3\%) of the participants were male. Over half of them (76.2\%) were either in the 1st or 2nd birth position. Four (14.8\%) had a sibling with similar problems, though most of them were in mainstream schools (73.9\%). Seventeen (58.6\%) were exclusively breast fed and seven admitted they had attended this screening exercise before, but for 21 (75.0\%) it was their first time (Table 3).

A comparison of those with autism and those with other neurodevelopmental problems showed that most of those with autism had given a history of deficits observed in social communicative behavior and restricted and repetitive interests. These reports were found to be statistically significant with reference to poor socializing, not seeming to listen when spoken to, and not having one good friend, insistence on sameness and lack of showing interest in others. All reported a higher occurrence in the ASD group than the other neurodevelopmental disorders group, with a significance level at $\mathrm{P}<0.005$ (Table 4).

\section{Discussion}

Neurodevelopmental disorders like ASD are of significant public health concern among African children. The public health burden of Autism and its long term impact when left untreated, are of great importance. Partnerships among different sectors can be useful tool in this regard. ${ }^{8}$ Collaboration for health care delivery can include for-profit (both informal and formal) and not-for-profit (NGOs, faith-based organizations, communitybased organizations) entities as well as a range of for-profit financial institutions. ${ }^{12}$ In caring for those with autism spectrum disorder, partnerships provide assistance in intervention where there is inadequate support. Viable options for collaboration/partnerships that have been described include: PrivatePublic partnerships; multidisciplinary team partnerships; inter-sectoral partnerships, such as health and education. ${ }^{13}$

This small study highlights the significant burden of autism spectrum disorders among children with neurodevelopmental problems in Lagos. A lack of understanding

Table 1. Diagnosis among participants.

\begin{tabular}{lcc} 
Diagnosis & Frequency & $\%$ \\
Down syndrome & 7 & 8.3 \\
Autism & 29 & 34.5 \\
\hline Attention deficit hyperactive disorder & 10 & 13.1 \\
Seizure disorder & 4 & 4.8 \\
\hline Language speech disorder & 16 & 19.0 \\
Other neurodevelopmental disorder & 2 & 2.4 \\
\hline Intellectual disability only & 7 & 8.3 \\
Cerebral palsy & 10 & 11.9 \\
\hline Learning disability & 8 & 9.5
\end{tabular}

Some participants had comorbidities as such warranting more than one diagnosis hence $n$. is $>85$.

Table 2. Social and developmental characteristics of child with autism.

\begin{tabular}{lcc} 
Characteristics & N. & $\%$ \\
Male gender & 23 & 79.3 \\
Birth position 1st or 2nd & 16 & 76.2 \\
\hline Attends mainstream school & 17 & 73.9 \\
Exclusively breastfeed & 17 & 58.6 \\
\hline Sibling with similar problem & & \\
$\quad$ Neurodevelopmental history & 4 & 14.8 \\
$\quad$ Length of pregnancy (term 9 months) & 26 & 92.9 \\
Cried immediately at birth & 20 & 71.4 \\
$\quad$ Age child walked before or at 1 year & 23 & 79.3 \\
$\quad$ Had history of seizures & 5 & 17.9 \\
Speech impaired or non verbal & 26 & 92.9 \\
$\quad$ Not verbal & 11 & 40.70 \\
\hline
\end{tabular}


about the disorder seems evident in the community as many individuals with other disorders were brought for screening for autism spectrum disorder. The Neurodevelopmental disorders seen were varied and spanned a variety of disorders. However a third of them were found to meet the diagnosis of ASD after clinical evaluation. This finding has been reported by Bakare and Munir where they concluded that up to a third of patients from clinical setting met the diagnosis of ASD. ${ }^{14}$

Challenges of late identification and diagnosis of autism is still a major problem in Africa. ${ }^{15}$ The late presentation and diagnosis have been linked to ignorance, stigma and lack of early screening or skilled personnel. A hospital-based population study in Nigeria found prevalence of ASD to be $0.08 \%{ }^{16}$ That figure the authors described was most likely influenced by low help seeking behavior for childhood neurodevelopmental disorders (NDD). Another study in Nigeria found $11.4 \%$ school children with intellectual disabilities in Nigeria. ${ }^{17}$ Taking a look at the data from these clinic and school-based studies and comparing them to those obtained in this study, a much higher prevalence is found (30\%). Though the populations studied were different, thus making our comparison to be done with caution, the difference in figures is however quite significant enough to suggest that there really may be a significant prevalence existing in the larger community population.

The children with autism have been described elsewhere to be often of male gender, with a high risk of similar disorder in a sibling. ${ }^{18}$ This is similar to our finding here in which we found more males meeting criteria, and over a tenth having a sibling with similar ASD-like symptoms.

There were a few comorbidities identified in this study and the type of comorbidities seen appears to be similar to that reported by other researchers in Africa. Comorbidities here were mainly ADHD (6.9\%) and seizure disorders (3.4\%). Diagnoses of ASD in Africa have been described to be rarely made without some intellectual disability, ${ }^{19,20}$ while other researchers have also documented common comorbidity of epilepsy with ASD. ${ }^{21}$

Neurodevelopmentally, there seemed to be few reported perinatal incidents, as most of our ASD participants were from term deliveries and were reported to have cried at birth (71.4\%). The main negative early childhood experiences included history of seizures in $17.9 \%$, speech and language problems in $92.9 \%$ and poor eye gaze in $48.3 \%$ (Table 2).

Language deficits were the most common identifier described in the persons with autism in this study. Our finding is also similar to that reported by other researchers where most of the children presenting to an orthodox clinical practice setting lacked or had limited expressive language abilities. ${ }^{20,21}$ Though language is no longer a core criteria needed to make a diagnosis of autism in the current DSM 5 , it is an important specifier which can be improved with early speech and language intervention. Bakare and Munir noted that this pattern of non-verbal ASD presentation might be connected to late presentation and diagnosis. ${ }^{20}$ This further underscores the point that there is a need for early screening and detection as this has been found to improve outcome and ameliorate the impact of these deficits. Other common complaints here were coordination problems, inattention, being dependent and poor socializing. These concerns highlight the limiting nature of the disorder together with the burden by caregivers (Table 2).

Comparing the ASD group with the non ASD group of attendees there is a clear difference around some features that were ASD specific in both groups. Items such as socialization, insistence of sameness, and showing interest in others all showed statistically significant differences with the ASD diagnosis group showing more impairment. This finding is not unexpected seeing that there is a need to meet the DSM 5 ASD criteria to meet diagnosis. Parental endorsement of these features further supports the clinical assessment findings (Table 4).

The good response of caregivers to this screening exercise and the magnitude of morbidity that was found reveal a possible treatment gap. This becomes particularly important in settings where there are inadequate resources to ensure the public health sector is able to get services to those that need them. A committed private sector with a not-for-profit organization (in-country or international partners) and researchers has the potential for great impact in raising awareness among the populace and strengthening capacity of incountry experts to care for these children and their families in a more holistic manner.

The above findings and the clinical challenges reported by these parents in caring for their child with autism emphasizes the need for further large scale community screening of children with developmental disorders in developing countries to clearly determine diagnosis and guide caregivers accordingly.

In view of the limited human resources and poorly coordinated services for autism and many neurodevelopmental disorders in many

Table 3. Common concerns identified by caregiver in child with autism (Based on parents own description/words).

\begin{tabular}{lcc} 
Concerns & Frequency & $\%$ \\
Speech problems or not talking & 29 & 100 \\
Very dependent and inability to use toilet & 7 & 25.0 \\
\hline Difficult/Abnormal behavior & 12 & 42.9 \\
Hyperactivity & 5 & 17.9 \\
\hline Attention problems & 10 & 35.7 \\
Academic problems & 7 & 25.0 \\
\hline Not socializing & 6 & 21.4 \\
Comorbidities present & 4 & 13.4 \\
\hline
\end{tabular}

Table 4. Comparing history of autism spectrum disorder (ASD) like symptoms in ASD group and other neurodevelopmental disorders (NDD) group.

\begin{tabular}{lccc} 
& ASD, N. (\%) & Other NDD, N. (\%) & P-value \\
Not socializing & $6(21.4)$ & $2(4.0)$ & 0.015 \\
Doesn't seem to listen or respond when spoken to & $22(75.9)$ & $28(50.9)$ & 0.027 \\
\hline Responds to social interaction & $21(72.4)$ & $33(62.3)$ & 0.53 \\
Has repetitive movements & $21(72.4)$ & $33(62.3)$ & 0.35 \\
\hline Insists on sameness & $16(64)$ & $17(36.2)$ & 0.024 \\
Responds when called & $21(77.8)$ & $48(88.9)$ & 0.185 \\
\hline Shows interest in others & $13(44.8)$ & $41(88.5)$ & 0.000 \\
Appropriate eye gaze & $15(51.7)$ & $18(34.6)$ & 0.133 \\
\hline Total & 29 & 56 & \\
\hline
\end{tabular}


communities within Nigeria, inclusive and innovative strategies must be explored to assist in creating awareness regarding such diagnosis and linkages to available services in the community. The absence of suitably qualified personnel in many heath institutions to make the diagnosis, the lack of funding for large epidemiologic surveys and the need for intervention, make an important case for the use of inter-sectoral collaboration or organizational collaboration as a strategy to address these challenges in resource poor communities. Parents of children with autism would need further support to engage in services and should be guided based on their need to the available service options in their immediate communities

\section{Significance for public health}

The long term burden associated with neurodevelopmental disorders like autism spectrum disorders can further be complicated by late detection and commencement of interventions. This challenge is particularly rife in many African countries with inadequate resources, such as skilled personnel, or inadequate and poorly coordinated services resource. There is a mounting need for innovative strategies in these settings that can make use of appropriate interagency/organizational partnerships with focus to ensuring regular screening and with relevant services delivered to those at risk and those identified with ASD.

\section{Limitations}

There are limitations in the small sample size and the sampling process of participation via the public service announcements. There may be subtle influences on the selection of participants that responded. As such our findings may not be directly representative of the larger general population. Lastly the non use of a standardized ASD diagnostic schedule due to lack of locally trained personnel as at the time of this data collection, may raise some questions as regards the reliability of using a clinical assessment only for ASD diagnosis in this study.

\section{Conclusions}

There is a clear need for regular screening and detection exercises to help with early identification and referral to services. Parents of children with neurodevelopmental disorders such as autism struggle with many complexities in their child's presentation and often need professional guidance to access available service options scattered in the community. In resource-challenged settings, institutional collaborations can be a viable option in executing screening and intervention programs for autism spectrum disorders.

\section{References}

1. American Psychiatric Association. Diagnostic and statistical manual of mental disorders. 5th ed. Arlington: American Psychiatric Association; 2013.

2. Hebert EB, Koulouglioti C. Parental beliefs about cause and course of their child's autism and outcomes of their beliefs: a review of the literature. Issues Compr Pediatr Nurs 2010;33:149-63.

3. Mandell D, Novak M. The role of culture in families' treatment decisions for children with autism spectrum disorders. Ment Retard Dev Disabil Res Rev 2005;11:110-5.

4. Tek S, Landa RJ. Differences in autism symptoms between minority and nonminority toddlers. J Autism Dev Disord 2012;42:1967-73.

5. Mandell DS, Listerud J, Levy SE, PintoMartin JA. Race differences in the age at diagnosis among Medicaid-eligible children with autism. J Am Acad Child Adolesc Psychiatry 2002;4:1447-53.

6. Bakare MO, Agomoh A0, Ebigbo PO, et al. Etiological explanation, treat-ability and preventability of childhood autism: a survey of Nigerian healthcare workers' opinion. Ann Gen Psychiatry 2009;8:6.

7. WHO. Strategic alliances: the role of civil society in health. Discussion Paper No. 1 CSI/2001/DP1. 2001.

8. WHO. Trade foreign policy, diplomacy and health. Public-private partnerships for health. 2016. Available from: http://www.who.int/trade/glossary/story077 len/

9. Robins D, Fein D, Barton M. The modified checklist for autism in toddlers (M-CHAT). Storrs: University of Connecticut; 1999.

10. Allison C, Williams J0, Scott F, et al. The Childhood Asperger Syndrome Test (CAST): test-retest reliability in a high scoring sample. Autism 2007;11:177-89.
11. Scott FJ, Baron-Cohen S, Bolton P, Brayne C. The CAST (Childhood Asperger Syndrome Test): preliminary development of a UK screen for mainstream primaryschool-age children. Autism. 2002;6:9-31.

12. Harding A. Partnerships with the private sector in health: what the international community can do to strengthen health systems in developing countries. Final report of the Private Sector Advisory Facility Working Group Center for Global Development. 2009. Center for Global Development. 1800 Massachusetts Ave. NW. Washington DC 20036. Available from: www.cgdev.org

13. Mankoski RE, Collins M, Ndosi NK, et al. Etiologies of autism in a case-series from Tanzania. J Autism Dev Disord 2006;36:1039-51.

14. Edwards B, Marks G. Autism research: a call for collaboration. N Z J Teachers Work 2009;6:129-35.

15. Bello-Mojeed MA, Bakare MO, Munir K. Identification of Autism Spectrum Disorders (ASD) in Africa: Need for Shifting Research and Public Health Focus. In: Patel VB, Preedy VR, Martin CR, eds. Comprehensive Guide to Autism. New York: Springer; 2013. pp. 2437-2453.

16. Bakare MO, Igwe MN, Odinka PC, Iteke 0. Neuropsychiatric diagnosis and psychotropic medication prescription patterns in a mental hospital-based child and adolescent psychiatric service in Nigeria. $\mathrm{J}$ Health Care Poor Underserved 2011;22:751-5.

17. Bakare MO, Ebigbo PO, Ubochi VN. Prevalence of autism spectrum disorders among Nigerian children with intellectual disability: a stopgap assessment. J Health Care Poor Underserved 2012;23:513-8.

18. Bakare M0, Munir KM. Autism spectrum disorders (ASD) in Africa: a perspective. Afr J Psychiatry 2011;14:208-10.

19. Werling D, Geschwind D. Sex differences in autism spectrum disorders. Curr Opin Neurol. 2013;26:146-53.

20. Bakare M0, Munir KM. Autism Spectrum Disorders in Africa. 2011. Available from: http:/www.intechopen.com/articles/show/t itle/autism-spectrum-disorders-in-africa.

21. Bello-Mojeed MA, Ogun OC, Omigbodun 00, Adewuya 0A. Late identification of autistic disorder in Nigeria: an illustration with 2 case reports. Nig J Psychiatry 2011;9:31-5. 